\title{
La hipertrigliceridemia leve a moderada parece incrementar también el riesgo de pancreatitis aguda
}

Mild-to-moderate hypertrigliceridemia may increase the risk of acute pancreatitis

\author{
Objetivos \\ Evaluar el riesgo de pancreatitis en la hipertrigliceridemia leve a \\ moderada (177 a $885 \mathrm{mg} / \mathrm{dl}$; 2 a $10 \mathrm{mmol} / \mathrm{L}$ ).
}

\section{Diseño, lugar y pacientes}

Cohorte prospectiva en población danesa, basada en dos estudios, el Copenhagen General Population Study entre 20032015 y Copenhagen City Heart Study iniciado en 1976 a 1978. Se incluyeron 116.550 adultos de 47 a 66 años con mediciones plasmáticas de triglicéridos y fueron seguidos durante un promedio de 6,7 años, hasta la aparición de un evento, muerte, emigración o finalización del estudio. Fueron excluidos aquellos que habían presentado previamente pancreatitis (PA) o infarto de miocardio (IAM).

\section{Medición de resultados principales}

Se evaluó la incidencia de PA e IAM en 6 grupos de participantes estratificados de acuerdo a su nivel de triglicéridos sin ayuno previo (< 89; 89 a 176; 177 a 265; 266 a $353 ; 354$ a 442 y $\geq 443$ $\mathrm{mg} / \mathrm{dl})$. Para el análisis multivariable se utilizó un modelo de regresión de riesgo proporcional de Cox.

\section{Resultados principales}

Se encontró un aumento significativo en la incidencia de PA e IAM en los pacientes con valores de triglicéridos $\geq 89 \mathrm{mg} / \mathrm{dl}$
Pedersen S, y col. JAMA. 2016;176(12):1834-42.

comparados con aquellos con $<89 \mathrm{mg} / \mathrm{dl}$. El riesgo de presentar PA fue directamente proporcional al valor de triglicéridos. Estos resultados continuaron siendo estadísticamente significativos luego de ajustar por las diferentes variables analizadas.

Tabla 1. Riesgo de PA e IAM en individuos con triglicéridos elevados comparados con aquellos con niveles menores a $89 \mathrm{mg} / \mathrm{dl}$

\begin{tabular}{c|c|c}
\hline $\begin{array}{c}\text { Nivel de triglicéridos } \\
\text { (mg/dl) sin ayuno }\end{array}$ & $\begin{array}{c}\text { Pancreatitis } \\
\mathbf{H R}^{*}(\mathbf{I C} \text { 95\%) }\end{array}$ & $\begin{array}{c}\text { IAM } \\
\mathbf{H R}^{*} \text { (IC 95\%) }\end{array}$ \\
\hline $\mathbf{8 9}$ a $\mathbf{~ 1 7 6}$ & $1,6(1$ a 2,6$)$ & $1,6(1,4$ a 1,9$)$ \\
\hline $\mathbf{1 7 7}$ a $\mathbf{2 6 5}$ & $2,3(1,3$ a 4$)$ & $2,2(1,9$ a 2,7$)$ \\
\hline 266 a 353 & $2,9(1,4$ a 5,9$)$ & $3,5(2,6$ a 4,1$)$ \\
\hline $\mathbf{3 5 4}$ a 442 & $3,9(1,5$ a 10$)$ & $2,8(2$ a 3,9$)$ \\
\hline$\geq \mathbf{4 4 3}$ & $8,7(3,7$ a 20$)$ & $3,4(2,4$ a 4,7$)$ \\
\hline
\end{tabular}

*Hazard Ratio ajustado por: edad, sexo, educación, tabaquismo, hipertensión, uso de estatinas, diabetes, IMC, consumo de alcohol y litiasis biliar.

\section{Conclusión}

La hipertrigliceridemia leve a moderada, evaluada sin ayuno previo, está asociada con mayor riesgo de presentar PA e IAM en la población general.

Fuente de financiamiento: Herlev and Gentofte Hospital and Copenhagen University Hospital.

\section{Comentario}

La PA es una patología prevalente y potencialmente fatal, las causas más frecuentes son la litiasis biliar y el alcohol, seguidas de la hipertrigliceridemia (1 a 4\%). Si bien la hipertrigliceridemia severa es una causa establecida de PA todavía no hay consenso sobre el valor de triglicéridos por encima del cual el riesgo está incrementado. Las guías del Colegio Americano de Gastroenterología ${ }^{1}$ y la Sociedad Americana de Endocrinología ${ }^{2}$ determinan que el punto de corte debería ser $1000 \mathrm{mg} / \mathrm{dl}$, la Sociedad Europea de Cardiología ${ }^{3}$ lo establece en $885 \mathrm{mg} / \mathrm{dl}$ y el ATP III $^{4}$ en $500 \mathrm{mg} / \mathrm{dl}$. Estos valores surgen de publicaciones en pacientes de alto riesgo. Debido a que el riesgo en la población general no está establecido y ante la falta de consenso en el punto de corte los autores quisieron evaluar si la hipertrigliceridemia levemoderada también está asociada a un mayor riesgo de presentar PA. Los resultados corroboraron la asociación lo cual podría modificar el manejo actual de la hipertrigliceridemia.

Vale destacar que las mediciones de triglicéridos fueron tomadas sin ayuno previo en concordancia con las recomendaciones de la Sociedad Danesa de Bioquímica Clínica. En 2016 la Sociedad
Europea de Ateroesclerosis publicó un consenso donde evalúa la medición del perfil lipídico sin ayuno y en base a numerosos estudios observacionales concluye que las muestras deberían tomarse sin ayuno previo debido a que se ha demostrado que no hay diferencias clínicamente significativas con las mediciones en ayunas para triglicéridos, colesterol total, colesterol LDL, colesterol remanente y colesterol no-HDL y sugiere tomar la muestra en ayunas cuando el valor de triglicéridos es mayor a $440 \mathrm{mg} / \mathrm{dl}$.

\section{Conclusiones del comentador}

Hasta el día de hoy, se consideraba que solo la hipertrigliceridemia severa estaba asociada a un mayor riesgo de PA basado en estudios en población de alto riesgo. El presente estudio muestra que en población general la hipertrigliceridemia levemoderada también está asociada a un mayor riesgo de presentar pancreatitis. Estos hallazgos deberían ser confirmados en futuros estudios para modificar el manejo actual de las hipertrigliceridemia leve-moderada.

María Florencia Decarlini [ Servicio de Medicina Familiar y Comunitaria del Hospital Italiano de Buenos Aires. maria.decarlini@ hospitalitaliano.org.ar]

Decarlini MF. La hipertrigliceridemia leve a moderada parece incrementar también el riesgo de pancreatitis aguda. Evid Act Pract Ambul. 2017;20(2):56. Comentario de Pedersen S y col. Nonfasting mild-to-moderate hypertrigliceridemia and risk of acute pancreatitis. JAMA Intern Med, 2016;176(12):1834-1842. PMID: 27820614.

\section{Referencias}

1.Tenner S, y col. American College of Gastroenterology. American College of Gastroenterology guideline: management of acute pancreatitis. Am $\mathrm{J}$ Gastroenterol. 2013;108(9):1400-1415, 1416

2. Berglund L, y col; Endocrine society. Evaluation and treatment of hypertriglyceridemia: an Endocrine Society clinical practice guideline.J Clin Endocrinol Metab. 2012;97 (9):2969-2989.

3. Catapano AL, y col; European Society of Cardiology (ESC); European Atherosclerosis Society (EAS). ESC/EAS Guidelines for the management of dyslipidaemias the Task Force for the management of dyslipidaemias of the European Society of Cardiology (ESC) and the European Atherosclerosis Society (EAS). Atherosclerosis. 2011;217(1):3-46. 4. National Cholesterol Education Program (NCEP) Expert Panel on Detection, Evaluation, and Treatment of High Blood Cholesterol in Adults (Adult Treatment Panel III). Third Report of the National Cholesterol Education Program (NCEP) Expert Panel on Detection, Evaluation, and Treatment of High Blood Cholesterol in Adults (Adult Treatment Panel Report of the National Cholesterol Education
III) final report. Circulation 2002; 106:3143. 